\title{
Interactive \& Multiscale Thematic Maps: A Preliminary Study
}

\author{
Robert E. Roth ${ }^{\mathrm{a}, *}$, Meghan Kelly ${ }^{\mathrm{a}}$, Nick Underwood ${ }^{\mathrm{a}}$, Nick Lally ${ }^{\mathrm{b}}$, Kristen Vincent ${ }^{\mathrm{c}}$, Carl Sack ${ }^{\mathrm{d}}$ \\ ${ }^{a}$ University of Wisconsin-Madison, CartographyLab(USA), reroth@wisc.edu,mkelly22@wisc.edu,nunderwood3@wisc.edu \\ ${ }^{b}$ University of Kentucky (USA), nicklally@uky.edu \\ ${ }^{c}$ City of De Pere, Wisconsin (USA) kristen.vincent@snc.edu \\ ${ }^{d}$ Fond du Lac Tribal \& Community College (USA), carl.sack@fdltcc.edu \\ * Corresponding author
}

\section{Keywords: Thematic Maps, Interactive Maps, Web Maps, User Studies, User Experience Design}

Problem: Interactive or "slippy" web maps have revolutionized cartography. Slippy maps present a single, coherentlydesigned reference map that can be panned to numerous geographic locations and zoomed across multiple scales. Further, they apply scale-dependent style rules to detailed geographic datasets, with the resulting designs rendered as a large set of interlocking tiles. To account for constraints in data bandwidth, processing, and storage, only those tiles relevant to the user's location and past interactions are served into the web browser or other application, resulting in a seamless, realtime user experience of "a map of everywhere". These slippy tilesets often are used as basemaps for advanced cartographic web and mobile applications, overlaying thematic information and other linework. Arguably, such slippy map mashups are the most common map seen and used today (and perhaps of all time). Yet, most of the cartographic design canon was developed long before slippy maps were possible. Do any of our time-tested design traditions in thematic cartography apply in today's interactive and multiscale mapping context? In this presentation, we discuss preliminary insights from an online map study about the design of interactive and multiscale thematic maps.

Background: Our research integrates the cartographic tradition in thematic representation with growing research on interaction design. Thematic maps depict the distribution of one or several geographic phenomena, with the base reference information used as context for interpreting spatial variation in the thematic information [1]. Thematic maps grew in popularity in the $19^{\text {th }}$ century at the onset of the modern global economy as a method for tracking and controlling materials, labor, and goods [2], and, in today's information-driven society, are created by cartographers for reasons ranging from popular news reporting to exploratory scientific visualization. Thematic maps enable geographic imagination and spatial thinking, often representing abstract or statistical concepts that cannot be observed directly. Common thematic map types include choropleth, dot density, proportional symbol, and isoline, among others [3].

Thematic maps can be organized in at least two ways. First, thematic map types differ in the visual variable used to encode the thematic information, or the basic graphic dimension separating variation in the thematic attribute of interest from other reference context [4]. For instance, choropleth maps typically employ the visual variables related to color (particularly color value, sometimes crossing multiple hues or saturations), dot density maps use a combination of arrangement and size, an emergent visual dimension sometimes described as "numerousness" [5], proportional symbol maps use size, and isoline maps use location (creating a new set of geographic linework based on thematic information), and also can include a color ramp between isolines similar to choropleth maps.

Second, thematic maps differ in the visual metaphor they evoke in the relationship between the thematic symbol and the reference context, such as an administrative unit for enumerated information. Common thematic map types vary in their congruency to geographic phenomena on two axes: discrete vs. continuous (i.e., how they exist in space) and abrupt vs. smooth (i.e., how they vary across space) [6]. Choropleth maps evoke a metaphor of continuous and abrupt phenomena (e.g., congruently matching governmental activities and policies like tax rates), proportional symbol evoke a discrete and abrupt metaphor (e.g., economic sites of production, like factories), dot density evoke a discrete and smooth metaphor (e.g., people and social phenomena), and isoline maps evoke a continuous and smooth metaphor (e.g., environmental or physical phenomena). The cartographic canon recommends taking into account both the visual variable and visual metaphor when selecting a thematic map for a particular thematic dataset (Figure 1).

Similar logics can be applied to organize cartographic interaction design and the mapping interfaces that support such interaction. The interactive dialogue between a user and a map mediated through a computing device can be deconstructed into interaction primitives, or the basic and observable units of interactivity [7]. From the user perspective, these include intentions or objectives for using the map and, from the map design perspective, these includes implementation of interactive functions or operators to manipulate the map [8]. Map-based objectives include feature identification, comparison, ranking, association, or delineation, while map-based operators include functions common to slippy web maps, like panning, zooming, and detail retrieval, and more complex functions such as filtering, reexpression, and sequencing, among others [9]. Each operator then is implemented using a graphic user interface (GUI) style, evoking visual metaphors for congruent interaction with analog objects to improve the user experience [10]. 
Method: We conducted an online study using the MapStudy experimental apparatus to address the above research questions following a 4x2 factorial design. MapStudy is an interactive map survey application developed in the UW-Madison Cartography Lab that allows for interaction logging in addition to quantitative and qualitative survey measures. We recruited 240 participants on Amazon Mechanical Turk, 30 per group in the $4 \times 2$ factorial study. Participants were compensated $\$ 3.50$ for participation, with the study requiring 25 minutes.

Experimental materials varied by thematic map type (Factor \#1: choropleth, dot density, proportional symbol, tinted isoline: see Figure 1 for examples) and interaction complexity (Factor \#2: slippy including pan and zoom, slippy+ including pan, zoom, and detail retrieval). Thematic map type (F1) was assigned within subjects, but interaction complexity (F2) was assigned between subjects to avoid participants learning operators that are later removed. The thematic maps depicted synthetic twitter data and were presented at two levels of resolution navigable through zooming: 25 square U.S. counties (overview) and 625 square U.S. townships (25 per county; details view). Accordingly, scale/resolution was an imbalanced Factor \#3 for analysis, self-selected by participants through zooming. We selected Western Kansas for a consistent geography and expected low familiarity with recruited users. Only the visual variables associated with the thematic map were varied, with tweets presented on an ordinal scale with five classes across map types. Other aspects of visual design were held constant.

Participants first completed a learning block introducing the provided operators, the legend design (but not the tested thematic symbolization), and the map reading tasks. Participants then viewed four "map reading" blocks showing different thematic map types (F1), with four trials for each block using the same thematic map type for a different synthetic tweet distribution (16 trials total). The order of blocks and distributions within blocks were balanced using Latin Squares. Each trial within a block varied in the user objective and target scale/resolution (F3): compare + details view, compare + overview, rank + details view, rank + overview. Participants completed the map reading trials while interacting with the map. Following the 16 map reading trials, participants then completed an "exploration" block in which they interacted with each thematic map type (F1) as a separate trial (four exploration trials in total) and then responded to recall cluster delineation objective and preference questions on a separate page without the interactive map. The study closed with a background survey. Map interactions were logged throughout.

Our map study design resulted in three independent variables (IDVs) by factor: thematic map type, interaction complexity, and scale/resolution. The design considered interaction effects by individual user differences collected from the background survey. IDVs and interaction effects then were evaluated by the following dependent variables (DVs): correctness (split by compare, rank, and delineate objectives), response time, self-reported confidence, self-reported difficulty, interaction sequence, and preference.

Outlook: We are continuing to collect participant responses from Mechanical Turk through January 2019 to reach our target of 240 participants. We anticipate the study to result in new guidance for interactive and multiscale map design as well as suggestions for future research at the intersection of thematic representation and interaction design. This research was funded by NSF CAREER \#1555267 and the Wisconsin Alumni Research Foundation.

\section{References:}

1. Tyner, J.A., Principles of map design. 2014: Guilford Publications.

2. Robinson, A.H., Early thematic mapping in the history of cartography. 1982.

3. Merson, J., Common Thematic Map Types, in The Geographic Information Science \& Technology Body of Knowledge, J.P. Wilson, Editor. forthcoming.

4. Bertin, J., Semiology of graphics: diagrams, networks, maps. University of Wisconsin Press, Madison, Wisconsin, $1967 \mid 1983$.

5. Nelson, E., The impact of bivariate symbol design on task performance in a map setting. Cartographica: The International Journal for Geographic Information and Geovisualization, 2000. 37(4): p. 61-78.

6. MacEachren, A.M. and D. DiBiase, Animated maps of aggregate data: Conceptual and practical problems. Cartography and Geographic Information Systems, 1991. 18(4): p. 221-229.

7. Roth, R.E., Cartographic interaction primitives: Framework and synthesis. The Cartographic Journal, 2012. 49(4): p. 376-395.

8. Norman, D.A., The design of everyday things. 1988, New York, NY: Basic Books. 257.

9. Roth, R.E., An empirically-derived taxonomy of interaction primitives for Interactive Cartography and Geovisualization. Transactions on Visualization \& Computer Graphics, 2013. 19(12): p. 2356-2365.

10. Shneiderman, B. and C. Plaisant, Designing the user interface: Strategies for effective human-computer interaction. 5th ed. 2010, Boston, MA: Addison-Wesley. 